\title{
AC 2009-2019: DEVELOPMENT AND ASSESSMENT OF A NOVEL SYSTEMS BIOENGINEERING COURSE INTEGRATING MODELING AND EXPERIMENTATION
}

\section{Timothy Allen, University of Virginia}

Dr. Timothy E. Allen is an Assistant Professor in the Department of Biomedical Engineering at the University of Virginia. He received a B.S.E. in Biomedical Engineering at Duke University and M.S. and Ph.D. degrees in Bioengineering at the University of California, San Diego. Dr. Allen's teaching activities include coordinating the undergraduate teaching labs and the Capstone Design sequence in the BME department at the University of Virginia, and his research interests are in the fields of computational systems biology and bioinformatics.

\section{Jeffrey Saucerman, University of Virginia}

Dr. Jeffrey J. Saucerman is an Assistant Professor in the Department of Biomedical Engineering at the University of Virginia. He received a B.S. in Engineering Science at Penn State, and M.S. and Ph.D. degrees in Bioengineering at the University of California, San Diego. Dr. Saucerman has developed and taught an undergraduate course in linear signals and systems. His research interests include modeling cardiac signaling networks and live cell imaging.

\section{Jason Papin, University of Virginia}

Dr. Jason A. Papin is an Assistant Professor in the Department of Biomedical Engineering at the University of Virginia. He received B.S., M.S., and Ph.D. degrees in Bioengineering at the University of California, San Diego. Dr. Papin has developed and taught an undergraduate course in Numerical Methods and Biological Modeling and a graduate course in Computational Systems Biology in collaboration with Dr. Peirce-Cottler. His research interests include systems biology in infectious disease and cancer.

\section{Shayn Peirce-Cottler, University of Virginia}

Dr. Shayn Peirce-Cottler is an Assistant Professor in the Department of Biomedical Engineering at the University of Virginia. She received a B.S. in Biomedical Engineering at The Johns Hopkins University and a Ph.D. in Biomedical Engineering at the University of Virginia. Dr. Peirce-Cottler's teaching activities have focused on developing and teaching the undergraduate Capstone Design course in the BME department at the University of Virginia, and her research is focused on using in vivo and computational models to elucidate the mechanisms of vascular differentiation and the efficacy of stem cell-based therapies for wound repair. 


\title{
Development and Assessment of a Novel Systems Bioengineering Course Integrating Modeling and Experimentation
}

\begin{abstract}
Advances in the biomedical sciences are becoming increasingly dependent upon the application of rigorous engineering principles to the study of biological systems. Most existing bioengineering curricula lack integrative courses that combine systems modeling approaches with biological "wet-lab" experimentation. We have thus developed an undergraduate senior elective course entitled "Systems Bioengineering Modeling and Experimentation" in the Department of Biomedical Engineering at the University of Virginia. The goal of this integrative course is to enhance undergraduate preparedness for industry and graduate study in the emerging field of Systems Biology by teaching systems concepts and methods in the context of experimental techniques. The course covers modeling and experimental approaches spanning multiple scales in biology, including subcellular dynamics, cellular networks, and multicellular patterning. Modern experimental approaches include live-cell imaging, gene microarrays, and in vitro angiogenesis assays. Since the field of Systems Biology is still rapidly evolving and currently ill defined, the particular educational benefits gained from the integrative pedagogical approach we have taken have not been established previously. To determine the efficacy of this course for achieving depth of knowledge and cognitive skills in the systems approach to biomedical engineering, we administered a summative assessment instrument to all senior undergraduates in the year the course was offered, both who were in enrolled in the course and who were not, with time points before and after the course was offered. In this paper, we describe the course structure and preliminary assessment from the first offering of this course. We also review the educational benefits and challenges associated with teaching systems biology concepts: e.g. complexity and simplification strategies, integration of biological information across spatial and temporal scales, resolving literature discrepancies in the context of a model, and parameter estimation and model validation. Initial assessment results indicate that this course provides a successful model for introducing undergraduates both to state-of-the-art techniques and to the central systems biology paradigm of iterative cycling between models and experiments.
\end{abstract}

\section{Introduction}

The field of biomedical engineering (BME), which is still a young field relative to the more "traditional" engineering disciplines, has been responsible for many high-impact biomedical advances (both clinically and in basic research) over the past 50-60 years ${ }^{1}$. However, over the last decade the field of BME has been significantly transformed by far-reaching new scientific and technological developments. The human genome has been sequenced ${ }^{2,3}$, the field of bioinformatics has generated powerful data annotation and database management tools ${ }^{4}$, diagnostic and imaging approaches are evolving at a rapid pace due to advances in molecular nanotechnology 5 , and computational power and capabilities are increasing exponentially every year. But the faster the pace of biomedical discovery, the more an integrated "systems approach" is needed to provide an accurate and useful context for those discoveries. It is now clear that taking such a "systems approach"-viewing not just the parts, but also the interactions 
between the parts and the structure of the system as a whole - is going to be necessary for making sustainable and significant impacts on human health ${ }^{6}$.

A systems approach to biomedical research requires knowledge of human physiology and pathology, in addition to quantitative skills in mathematics and engineering ${ }^{7}$. More importantly, however, it requires the ability to integrate these subjects in a meaningful way ${ }^{8}$. Biomedical engineers, who already receive training in biology/physiology and the quantitative sciences, are uniquely poised to learn, apply, and evolve the integrative systems approach, and in so doing, further basic medical discovery and innovate new solutions for human health through applied research and technology development ${ }^{6}$. But biomedical engineering curricula nationwide have, to date, largely neglected the development of integrative courses that combine experimental and systems methodologies in a multi-scale fashion (i.e. subcellular, cellular, and tissue-level). While a number of BME undergraduate programs currently offer elective courses in bioinformatics, sequence analysis, and/or mathematical modeling in BME, none to our knowledge combine "wet-lab" high-throughput data generation with both data analysis and integrated large-scale models of biological systems.

We have therefore developed a one-semester undergraduate elective course for upper-level biomedical engineers at the University of Virginia that explicitly teaches systems bioengineering methodologies and concepts through collaborative and immersive high-throughput wet-lab experiments and computational analyses that span the different spatial scales in biology-from the DNA-level through the organ-level. Within both the biotechnology sector and in academia, the demand for graduates who possess expertise in the generation of high-throughput data - as well as the modeling skills needed to analyze and predict pathological states and identify viable therapies - has increased dramatically ${ }^{9}$. One key objective of this course is thus to prepare BME graduates for working in a rapidly evolving and interdisciplinary field that requires not only new experimental and computational tools, but also a new "systems" way of thinking and problemsolving in healthcare and medicine.

\section{Prerequisites}

Given the topics covered in the Systems Bioengineering Modeling and Experimentation course developed at the University of Virginia, it was designed as a senior elective course such that the students had sufficient background in computational modeling, systems analysis, and experimental biological techniques. Thus, the prerequisites are a molecular biology laboratory, engineering systems analysis (and its prerequisites, including multivariate calculus, differential equations, and statistics), and aptitude in computational methods (e.g. Matlab programming) necessary to execute the modeling portion of the course. These requirements are typically satisfied by most BME programs by the $3^{\text {rd }}$ or $4^{\text {th }}$ year of study in the undergraduate curricula.

\section{Course Structure}

Towards addressing the growing demand for BME graduates who possess the skills and understanding required to contribute to the generation and systems analysis of high-throughput data, we have established a significant systems bioengineering facet within the undergraduate major in Biomedical Engineering at the University of Virginia. The new systems bioengineering 
course within the BME major at U.Va., intended for the fourth year of study, covers concepts spanning multiple scales in biology, from intracellular to multicellular systems. Accordingly, the course is divided into three modules (Figure 1):

- $\quad$ Module 1 - Subcellular dynamics: Modeling and analysis of dynamic biochemical processes that regulate cell function, as well as the experiments and image analysis necessary to generate such data.

- Module 2-Cell-scale modeling and gene expression: Generation and analysis of highthroughput gene expression data. Standard microarray data analysis methods were covered. In addition, cell-scale model formulation and analysis were taught, and modeling projects were integrated with representative high-throughput data.

- $\quad$ Module 3 - Multicellular behavior: Studying multi-cell tissues that are spatially and temporally complex using agent-based modeling in combination with in vivo experimental manipulations.

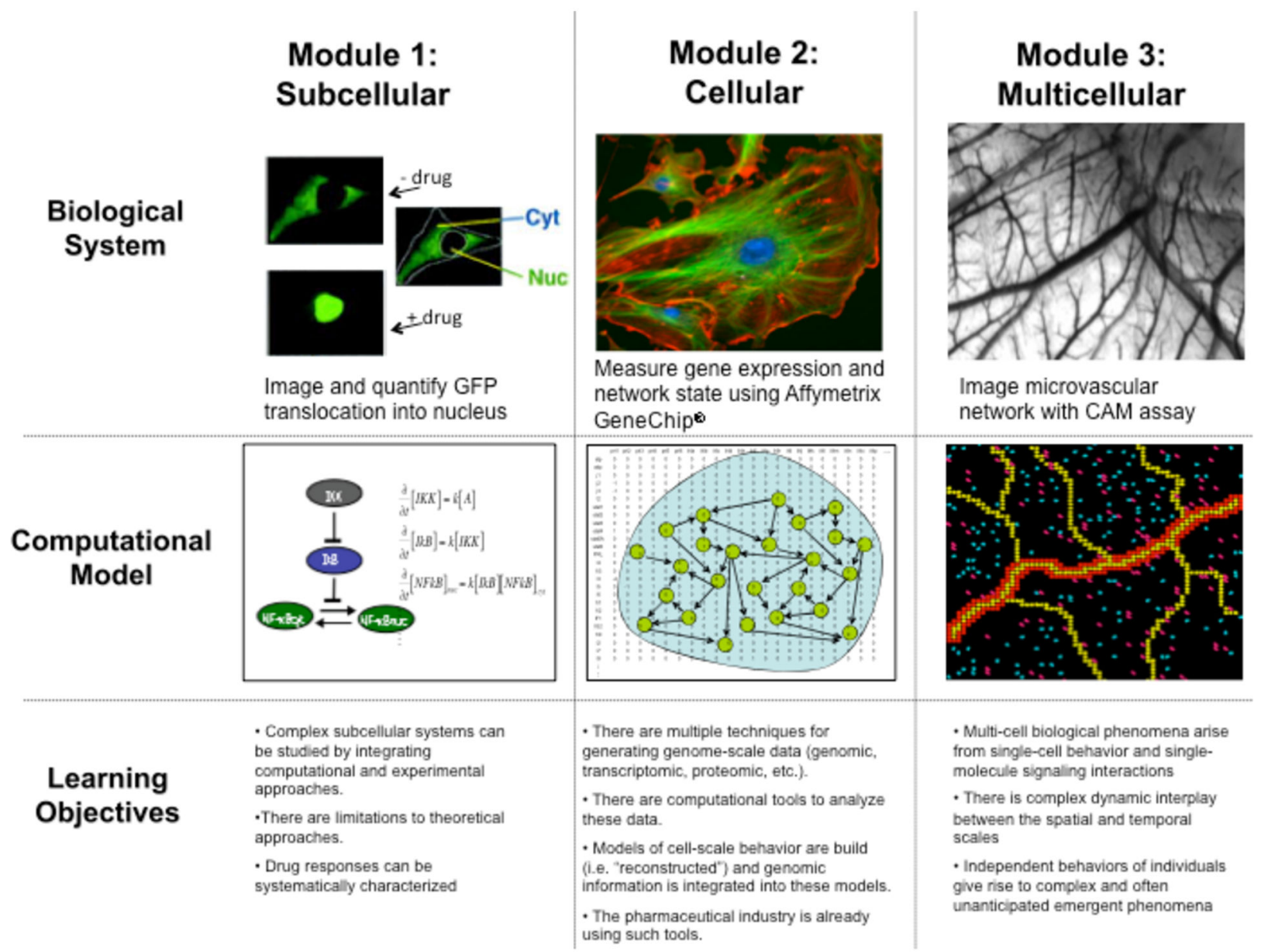

Figure 1. Modular organization of Systems Bioengineering elective course highlighting the learning objectives of each module and the key linkages of experiments with modeling. 
Since there was a wide breadth of material covered in this course, we necessarily had to be very selective about the material covered in each module, so that students had an opportunity to gain an appreciation for the issues and limitations of the most common modeling and experimental approaches at each scale. Each of the three modules in the course had the following general goals in common:

1. Introduce an especially medically relevant problem (in lecture and by assigning review papers to read) such that the students learn the motivation/need for a systems approach to solving this problem.

2. Lay the theoretical foundation: In lecture, we reviewed the underlying physical principles (linking back to material taught in prerequisite courses), specific modeling methodologies to describe these underlying principles, and the theory behind experimentally testing such models.

3. Wet-lab experiments: The specific skills required to generate the data needed to properly validate a systems model was taught within a hands-on laboratory.

4. Data analysis and integration with the previously discussed model (or just previously mentioned rather than discussed, depending on the module). Students had to link their results and analysis to the initially identified medically relevant problem and also suggest next steps.

5. Assess students' ability to synthesize all aspects of the module by having them undergo an oral examination explaining their approach, describing their results and analysis, and discussing the implications. Key concepts were also tested on a written final exam at the end of the semester covering all three modules. (See section on "Assessment of Student Learning" later in this paper for more specific information and rubrics relating to the oral exams and final written exam.)

\section{Rationale and Specific Content of Course Modules}

The integrative Systems Bioengineering Modeling and Experimentation course instituted at U.Va. was split into three topical modules, each of which covered a different scale and modeling framework. We began the course with a 3-lecture introduction to modeling fundamentals. Each of the three topical modules then lasted 3 weeks each (two 50-minute lectures per week). Additionally, Modules 1 and 3 contained two three-hour lab periods, and Module 2 contained one lab. In summary, Module 1 focused on the sub-cellular scale (i.e. DNA/protein), combining live-cell fluorescence imaging data with dynamic models of protein transport and cell signaling processes. Module 2 focused on the cellular scale and emphasized network modeling and the expression of the genome in the context of this overarching network. Module 3 covered tissuelevel models that rely on agent-based models, integrating these models with experimental data of a microvascular network in the chick embryo (derived from chicken eggs). Lectures were also given at transition points between modules and at the end of the semester to connect the different modeling scales and strategies, providing a cohesive picture of the modeling strategies most appropriate for the types of biomedical and biological questions that were addressed. 
Module \#1: Modeling and analysis of dynamic biochemical processes that regulate cell function, as well as the experiments and image analysis necessary to generate data. (Figure 2)

\section{1) Biomedical problem:}

The complex behaviors of the cell are regulated not by a central controller, but by distributed networks of biochemical reactions. Some biochemical networks (cell signaling networks) probe the extracellular environment for hormones and drugs, and then perform information processing to compute desired cell responses. Other biochemical networks (gene regulatory networks) regulate gene expression and thus the biological composition of the cell. Together, cell signaling and gene regulatory networks are responsible for an array of biological adaptations, particularly those changes seen in disease settings. Heart failure results not from the primary injury of a heart attack, but from abnormal gene expression and growth in response to the injury. Cancer kills not from tobacco or radiation per se, but from the damage to the biochemical control networks that keep tissue growth within reasonable limits.

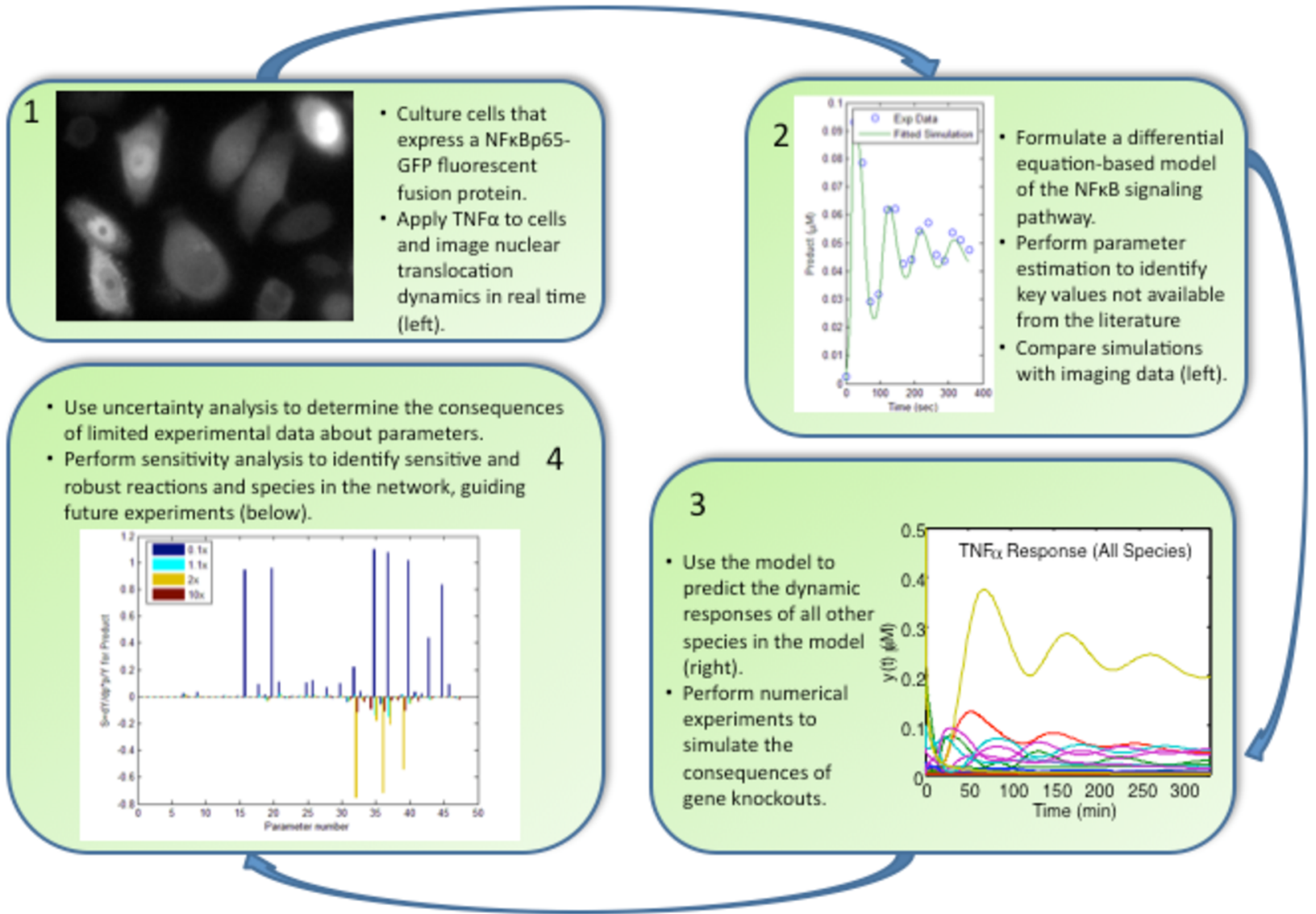

Figure 2. Iterative nature of experiments and network analysis performed in Module 1 of the Systems Bioengineering Modeling and Experimentation course. The images were created by actual student teams who took the course in Fall, 2008.

As a result, the vast majority of drugs target these biochemical control networks. Over $50 \%$ of prescriptions drugs are aimed at just one class of biochemical receptors alone ${ }^{10}$. Both academia 
and industry have now recognized the need for systems approaches, both computational and experimental, for understanding and treating such complex networks. Recent large-scale academic efforts such as the Alliance for Cellular Signaling and the Cell Migration Consortium have identified systems modeling as essential for a comprehensive understanding of signaling networks and the response to genetic and pharmacological perturbations ${ }^{11,12}$. Large biotech and pharmaceutical companies such as Novartis, Pfizer, and Bayer have now established Systems Biology or Modeling departments and are developing dynamic computational models to understand the responses of drugs in these complex cellular environments ${ }^{13}$. Rather than relying on test-tube biochemistry where results may be taken out of context, small and large firms alike (including Pfizer, Johnson and Johnson, GlaxoSmithKline, Cellomics, Bioseek) are now turning to cell-based assays where drugs are tested in the intact cellular environment ${ }^{14}$. Thus both computational and experimental systems approaches are greatly needed to understand complex disease mechanisms and identify appropriate therapeutic strategies.

\section{2) Specific modeling concepts covered:}

Modeling dynamic biochemical processes: Differential equations are the foundation for dynamic analysis in the physical sciences and engineering, where models are based on physical conservation laws. While systems analysis using ordinary and partial differential equations is taught in all biomedical engineering curricula and in many other fields (and these approaches are prerequisites for the proposed course), several aspects are not typically taught at the undergraduate level but are vital to analysis of biological systems. For example, biology is highly nonlinear, and biological parameters and even mechanisms often have a large degree of uncertainty ${ }^{15}$. As a result, purely "bottom-up" approaches often fail, and models must be iteratively refined by comparison with experimental data. In models of complex, heterogeneous networks such as gene regulation, it may be difficult to determine which components or interactions are most important for a given behavior. Therefore students were taught:

- How to mechanistically model biochemical reaction networks with nonlinear differential equations, emphasizing differences from linear systems;

- How to quantitatively validate models using experimental data and discriminate among competing hypotheses/models;

- Parameter estimation approaches for developing models based on experimental data, including measures of uncertainty; and

- Parameter sensitivity analyses to identify critical components/interactions.

3) Specific experimental skill sets learned:

Fluorescence microscopy approaches, particularly those involving genetically tagged proteins like green fluorescent protein (GFP), have revolutionized our ability to quantitatively measure biochemical dynamics in intact cells and organisms ${ }^{5}$. Such cell-based assays based on fluorescence imaging of biochemical activity and translocation are used in a variety of drug discovery platforms used in firms like Cellomics, BD Biosciences, and Pfizer. In the proposed course, students complemented computational modeling by imaging dynamic subcellular responses to drugs. Students learned: 
- How to image subcellular translocation of GFP-labeled proteins in response to drugs like TNA $\alpha$ (tumor necrosis factor - alpha) using fluorescence microscopy;

- How to process images to quantify subcellular dynamics and drug efficacy (using the ImageJ software package); and

- How to integrate imaging data with models for parameter estimation or model validation (using Matlab).

4) Particular insights or general cognitive growth that students experienced in this module:

Students learned how to analyze and understand complex subcellular systems by integrating computational and experimental approaches (Figure 2). By doing so, students better appreciated the complementary roles of modeling (e.g. hypothesis generation, quantitative analysis, etc.) and experiments (hypothesis testing), limitations of theoretical approaches, and how drug responses were systematically characterized. Practical issues regarding appropriately dealing with noisy data were discussed, particular with respect to normalization and quantification of protein translocation to be included as model inputs.

Module \#2: Reconstruction and analysis of cell-scale networks. (Figure 3)

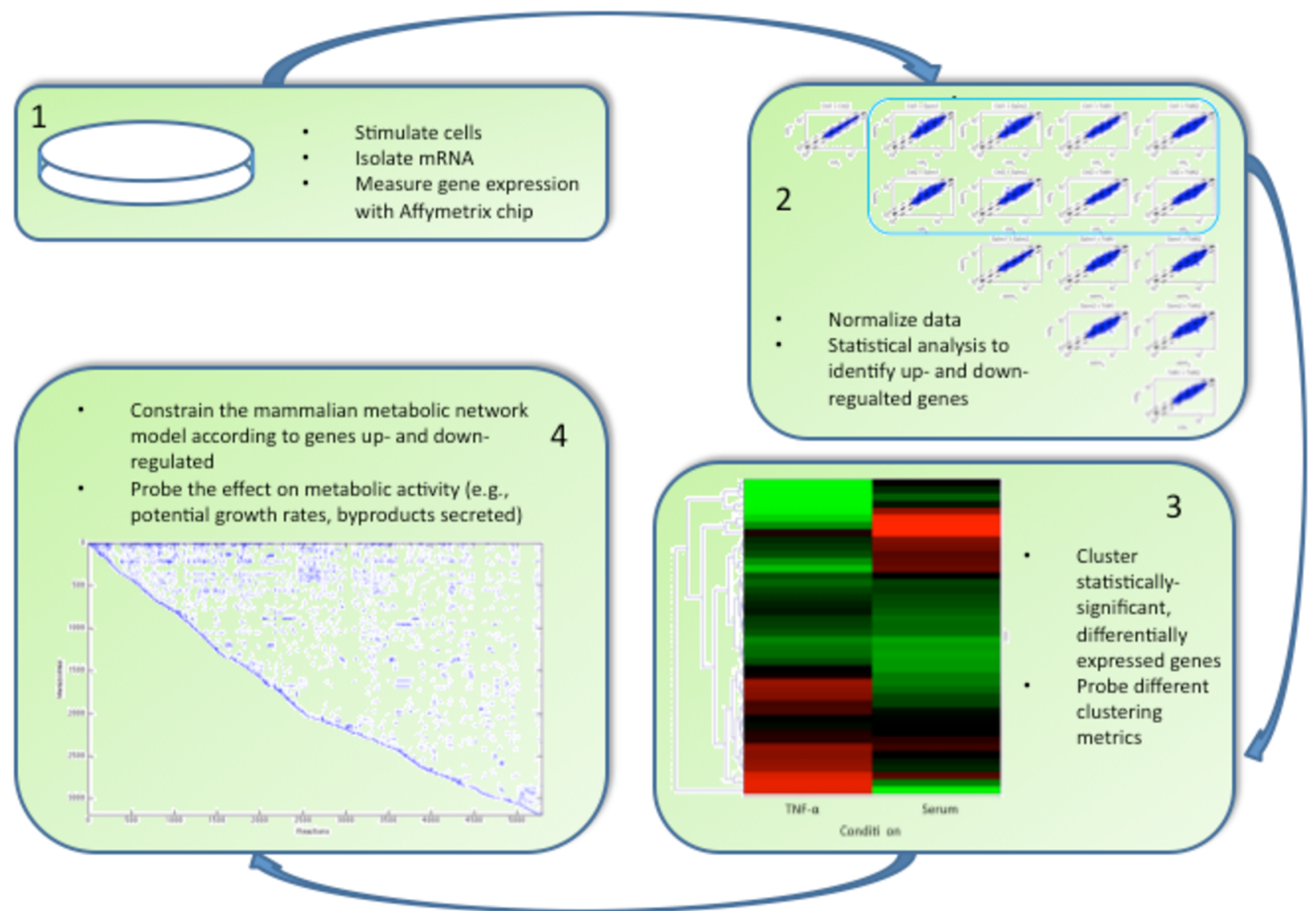

Figure 3. Iterative nature of experiments and network analysis performed in Module 2 of the Systems Bioengineering Modeling and Experimentation course. The images were created by actual student teams who took the course in Fall, 2008. 


\section{1) Biomedical problem:}

The wealth of cell-scale or genomic information necessitates computational approaches that can deal with uncertainty in the data and yet provide testable hypotheses of cell physiology. Multiple genomes have been sequenced and annotated, and experimental technologies are providing genomic, proteomic, transcriptomic, and other cell-level data sets. Paralleling the development of these experimental technologies are computational methods for analyzing these data sets and generating models of cell function. While dynamics models described above can be particularly informative for an evaluation of time courses for signaling and regulatory pathways, such parameters are unavailable for large-scale systems. An analysis of a genome-scale system can provide hypotheses for which components to target in a drug discovery program that may be further refined with the skills taught in Module 1.

\section{2) Specific modeling concepts covered:}

Statistical analysis of gene expression data: With the gene array data generated in the course, the students learned basic principles of statistical analysis of the data, which are widely applicable to other technologies. The students learned to appreciate normalization of the data and similarity measures. The students also analyzed the data with a variety of clustering approaches (e.g., K-means, SVD, hierarchical). We also discussed case studies in which pharmaceutical companies and academic laboratories have used such approaches to generate medically relevant signatures of disease (e.g. see the recent FDA approved marker of breast cancer recurrence comprised of 70 gene expression states ${ }^{16}$ ).

Topological modeling approaches: Building off the dynamic analysis methods learned in Module 1, students appreciated the additional challenges in large-scale modeling efforts for which large sets of dynamic parameters are not available. Students were provided with an overview of modeling approaches to study and characterize network topology for studying metabolic, regulatory, and signaling networks ${ }^{17-19}$. Modeling skills derived from linear algebra, linear programming, graph theory, and other similar approaches that have been used to study properties of such networks were covered within the module.

\section{3) Specific experimental skill sets learned:}

Gene expression microarrays have become standard tools in the pharmaceutical industry to probe system-wide responses to drug action, among a multitude of other applications ${ }^{20}$. Students isolated RNA from bovine arterial endothelial cells (BAECs) under treatment with TNF $\alpha$, under serum stimulation, and from a control population with no growth factor, and they prepared the RNA samples for hybridization. The Biomolecular Research Facility at U.Va. generated the expression profiles using the bovine genome GeneChip arrays (Affymetrix, Inc.). The students were given a tour of the core facility, and the technician who performs the chip processing identified the equipment and its respective contribution to the process from isolated RNA to the gene expression data that the students analyzed.

4) Particular insights or general cognitive growth that students experienced in this module: 
Students learned how genomic data (i.e. transcriptomic, proteomic) are generated, as well as the theory underlying such technologies. They learned to appreciate the computational tools that are used to analyze such data. Students understood how models are built of cell-scale behavior and how such genomic data are integrated into these models (Figure 3). These skill sets are being used in the pharmaceutical industry and thus case studies were presented to provide a context and motivation for learning such skill sets. ${ }^{20}$

Module \#3: Studying multi-cell tissues that are spatially and temporally complex using agentbased modeling in combination with in vivo experimental manipulations. (Figure 4)

\section{1) Biomedical problem:}

There is ample evidence that drug targets identified in cellular and subcellular analyses are not always relevant once evaluated in a multicellular context. Multiple physiological processes are a function of the interactions between cells and not simply the interactions between components in a cell. For example, every tissue in the body is dependent on the microvascular system (the smallest blood vessels in the body, including the capillaries) to deliver oxygen and nutrients. In healthy tissues, when the demand for oxygen and nutrients changes, or increases as in the case of an exercised bicep, the microvascular system can structurally and dynamically adapt ("remodel") by growing (sprouting) new blood vessels to meet the demand of the tissue ${ }^{21}$. These microvascular adaptations are necessary for delivering and maintaining an adequate blood supply, but when a tissue is diseased or injured, as is the case in a diabetic ulcer or a diseased heart, the natural remodeling response of the microcirculation may become compromised. In such pathological settings, an inadequate microvascular remodeling response can actually augment and accelerate the disease process - creating a downward spiral of an injured tissue unable to obtain enough oxygen and nutrients to repair itself, ultimately causing total tissue death that can manifest as a severe and life-threatening event in the organism (e.g. a heart attack). ${ }^{22} \mathrm{~A}$ viable therapeutic strategy, therefore, is to identify treatments (e.g. drugs, therapeutic stem cells, targeted gene therapy) aimed at enhancing or inducing microvascular growth and remodeling in settings of disease. ${ }^{23}$ However, microvascular growth and remodeling are very complex processes that involve many different cell types, cell behaviors, and biochemical and biomechanical signals. Even using our most sophisticated microscopes and imaging software, experiments alone do not facilitate the spatial and temporal resolution needed for understanding the complexities of microvascular growth and remodeling. Computational modeling alone is incapable of reproducing phenomena that are biologically realistic due to inadequacies in parameter estimation. To make sense of the complexity so that we can identify effective and safe targets for therapeutic manipulation, we must develop computational tools in tandem with experimental models, because only a dual approach is capable of providing such a systems-level analysis of tissues ${ }^{24}$. Teaching the experimental skills in the same context as teaching those skills needed to construct and utilize computational models is, therefore, invaluable to enabling students to make advances in computational bioengineering. In this module we aimed to teach students agent-based modeling and the experimental skills necessary to investigate dosing regimens of vascular endothelial growth factors (VEGF) in an in vivo model of microvascular growth with the goal of designing an effective strategy for therapeutic angiogenesis. 


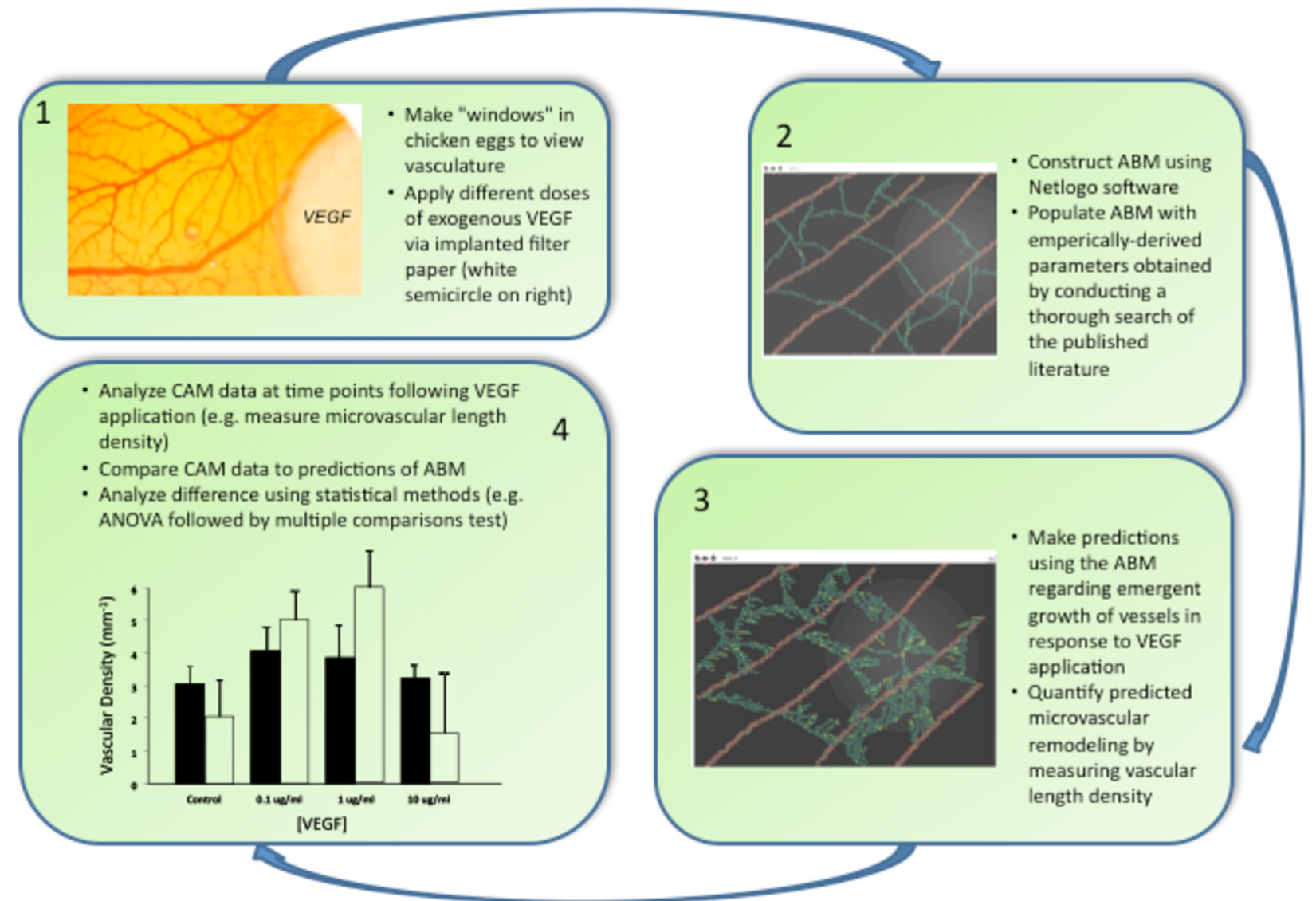

Figure 4. Iterative nature of using in vivo experiments (via the CAM assay for imaging microvessel growth) to validate and provide inputs for agent-based multicellular models. The images shown were generated by actual student teams from Fall, 2008.

\section{2) Specific modeling concepts covered:}

Agent-based modeling is a computational approach whereby individuals in a system (e.g. biological cells) and their interactions with one another are simulated within the spatially and temporally dynamic environment (e.g. biological tissue). The outcome is characterized as "emergent behavior," such as the growth of a new microvessel. The relationship between the rules that agents are instructed to follow and the emergent output at the multi-agent, or multi-cell scale, is an indication of how the system operates as a whole. Moreover, the agent-based model can be interrogated to identify bottlenecks in the system or key targets for intervention, which is useful for identifying new drugs or therapies to induce, for example, microvascular growth in the setting of heart disease ${ }^{25}$. With regards to the computational analysis emphasized in this module, the expected learning outcomes included:

- Understanding the underlying philosophy of agent-based modeling;

- Understanding how agent-based modeling has previously been used to simulate and study biological processes and tissue patterning phenomena, specifically;

- Knowing how to design and program agent-based models (specifically using NetLogo), and specifically how to design and program an agent-based model of microvascular growth and remodeling; 
- Knowing how to identify empirically-derived rules for agent behaviors and perform parameter estimation, when necessary;

- Appreciating the limitations and caveats of agent-based modeling;

- Having the ability to use agent-based models to derive new hypotheses and test alternative hypotheses.

3) Specific experimental skill sets learned:

The chick chorioallantoic membrane (CAM) assay of microvascular growth and remodeling is one of the most widely used experimental models for studying how the microvasculature grows and adapts in response to pharmacological manipulation (refer to right-hand panel of Figure 1 and to Figure 4). ${ }^{26-28}$ It is therefore considered an established method for high-throughput testing of drugs designed to stimulate microvascular growth. In this module, the learning outcomes for the experimental portion included the following:

- Knowing how to perform the microsurgery required for the CAM assay;

- Knowing how to deliver drugs in vivo to impact microvascular growth;

- Knowing how to use intravital microscopy to image the tissue;

- Knowing how to use image analysis software (specifically ImageJ) to quantitatively assess the amount of microvascular growth and remodeling resulting from the pharmacological intervention;

- Knowing how to use the experimental data to validate predictions of the agent-based model and to test hypotheses suggested by the computational model.

4) Particular insights or general cognitive growth that students experienced in this module:

Students learned how multi-cell biological phenomena arise from single-cell behavior and single-molecule signaling interactions, and the complex dynamic interplay between the spatial and temporal scales. They learned to appreciate how independent behaviors of individuals give rise to complex and often unanticipated emergent phenomena, and how, in the microcirculation, this paradigm relates to various disease states.

By building their own agent-based model of angiogenesis, students learned how to simplify this complex biological process and use a model to ask and answer relevant questions, such as the impact of dose response of an exogenously applied growth factor (VEGF). By acquiring their own data in the laboratory with the CAM assay and using these data to independently construct and subsequently validate the predictions of their model, the students had first-hand experience integrating experimental data with computational modeling. In so doing, the students appreciated the difference between "data-in" and "data-out" and the necessity of keeping the two data sets separate, so as to avoid simple curve fitting and truly make independent predictions with their model. This gave them a better appreciation for how the two approaches (experimental and computational) unite to accelerate the discovery process and enhance understanding of this complex system. 


\section{Assessment of Student Learning}

The Systems Bioengineering Modeling and Experimentation course was offered for the first time during the Fall 2008 semester at the University of Virginia within the Department of Biomedical Engineering. Nine students, all in their senior year as undergraduates in the BME major, enrolled in the course. Students worked in teams of three performing both the laboratories and the modeling and analysis for each module (three teams total). The bulk of the grade for each student came from three oral "mini-comprehensive" exams and a final written exam.

Oral "mini-comprehensive" exams: The small size of the class $(N=9)$ afforded us the opportunity to evaluate the students using an oral exam format. Each of the three modules concluded with an oral midterm exam, which served in lieu of a written midterm or module lab report. Since students in our program already receive extensive experience writing lab reports and giving formal presentations in other classes throughout the major, we felt that an oral exam format would be the optimal method by which to truly assess each student team's understanding of the module. These exams, which lasted 45 minutes each, gave the course instructors ample opportunity to ask in-depth questions and to especially probe wherever we spotted specific deficiencies or errors of understanding. The oral format also allowed us to help the students think through the problem in ways they may not otherwise have been able to do. Since these exams were conducted with each team of three rather than with the individual students, we would occasionally target questions to specific students (e.g. if they had been quieter during the exam or had earlier revealed a lack of knowledge in any particular area).

We developed a simple rubric for assessing student performance on the oral midterms, rating each of them in the following five areas: communication, knowledge, answering questions, accomplishment, and learning. The "communication" score reflected the students' ability to explain what they did in lab and in the post-lab modeling and analysis, as well as how clearly and crisply they defined terms and techniques. "Knowledge" was determined by their command of the relevant background information and modeling approaches. The score for "answering questions" reflected not only whether a first answer to a question was correct, but also how well the students were able to "think on their feet" when we asked follow-up questions and attempted to guide them to a greater understanding of a concept if they were initially deficient.

"Accomplishment" reflected the overall level of effort and work that went into their modeling and analysis over the module (assuming the work was correctly done), and "learning" was an overarching score that took into account the students' ability to synthesize their knowledge and the techniques they applied in the module to truly understand not only what they did, but why it was important. All 9 students did well on these oral midterms and demonstrated solid understanding of the labs, techniques, and underlying concepts (grades ranging from B to A+).

Final Written Examination: At the end of the semester, enrolled students took a three-hour written examination which not only covered all three modules, but also asked the students to integrate modeling approaches from among the three scales to demonstrate their appreciation for the challenges and possibilities associated with multi-scale modeling of biological systems. While a few of the exam questions asked students to recall definitions and explain specific concepts, most of the exam was very integrative and applied in nature, thus assessing higherorder cognitive skills identified on Bloom's Taxonomy ${ }^{2,30}$, specifically analyzing, evaluating, 
and creating (i.e. synthesis). For example, on the final exam, students were shown a recently published agent-based model of a biological process (and the associated set of "rules" governing that model), and they were also given figures showing experimental data generated to validate the model. Students had to evaluate whether or not the model made sense (given the data) and how they might improve the model. Towards addressing the synthesis/creating level on Bloom's Taxonomy, students then had to generate their own "data" (creating labeled figures or realistic results and sketching them on the exam paper) and to hypothesize model components (i.e. rules) that would account for these data.

Student performance on the final exam varied much more widely than on the oral midterms, ranging from $72 \%$ to $95 \%$ (average of $84 \%$ ). Given the difficulty of the exam (both in terms of thought required and of sheer length), the instructors were generally pleased with how the students met the learning outcomes on Modules 1 and 3. Six of the nine students underperformed on Module 2, leading us to recognize that certain concepts pertaining to highthroughout experimental techniques and network modeling (especially linking these two topics) needed to be given greater attention in the next iteration of the course to be taught in Fall 2009.

\section{Course Assessment}

One of our primary goals in assessing the Systems Bioengineering Modeling and Experimentation course was to determine the efficacy of this course for achieving not only depth of knowledge and cognitive skills in the systems approach to biomedical engineering, but also students' self-perceived abilities and confidence in the field to stimulate their interest in systems bioengineering. Summative assessment instruments were developed and administered both to Biomedical Engineering majors who did and who did not take the course during its first offering in Fall, 2008. This assessment was conducted at the beginning of the course (August 2008) and at the start of the semester following the end of the course (January 2009). The former served as a baseline measure of understanding and comfort level, and the latter tested for retention, since having met course objectives by any measure does not necessarily mean that the knowledge or skills will be effectively retained for future application. Those students who did not take the course were still subjected to requisite laboratory and computational courses that are part of the major program of study in BME. They therefore constituted an appropriate control group since they were exposed to much of the same fundamental knowledge in biology and mathematics, but without the fully integrated or contextualized nature of systems bioengineering.

Both survey instruments consisted of the following 14 questions:

1. Suppose that your first job is for a small biotech/pharmaceutical company. Just a few weeks into your job, your supervisor asks you to use an existing computational model to identify the best potential drug targets within a metabolic network. Rate your current confidence level at accomplishing this task on a scale of 1-5 (5 being "extremely high confidence").

2. You are asked to write Matlab code that solves a system of 20 ordinary differential equations (ODEs) that are hypothesized to describe the behavior of a well-characterized cell signaling network. Although this particular network has never been mathematically modeled before, the specific kinetic parameters have been determined in previous studies. 
Rate your current confidence level at accomplishing this task on a scale of 1-5 (5 being "extremely high confidence").

3. In your first year in graduate school, suppose you are asked to use microarray data (total of 3,217 genes probed) to detect which genes are up-regulated or down-regulated following stimulus with a specific growth factor. On a scale of 1-5, rate your current level of confidence in your ability to apply appropriate statistics toward carrying out this task (5 being "extremely high confidence").

4. Atherosclerosis is a complex disease that involves fluid flow forces impacting endothelial cell adhesion, diffusible circulating chemokines impacting inflammatory cell trafficking, smooth muscle cell production of extracellular matrix modulators, and lipoprotein metabolism by macrophages and foam cells in the plaque. Each of these processes results in innumerable signaling events that, as an ensemble, cause a plaque to enlarge over time. On a scale of 1-5, rate your confidence level in your ability to integrate these diverse processes and signals into a unified model so that you can test the effect of a particular chemokine inhibitor on the enlargement of an atherosclerotic plaque (5 being "extremely high confidence").

5. Are you confident in your ability to break a large modeling problem into smaller, more manageable pieces? Rate your current confidence level at accomplishing this task on a scale of 1-5 (5 being "extremely high confidence").

6. Suppose your supervisor gives you one gene expression (microarray) data set taken from cells isolated from a cirrhotic human liver. Your supervisor then asks you to use this data to identify the genes that would be the best targets for a drug that will reverse the effects of cirrhosis. Would you feel comfortable working on this problem using this data?

7. Suppose you are asked to teach/mentor a biological modeling approach to someone who already has a solid background in mathematics, cell biology, and physiology. You must provide your "student" with the known mechanisms of a particular disease (with which you yourself are already very familiar), and then teach this person to determine the appropriate length scale at which to model the disease process (e.g. cellular, molecular, tissue, etc.) and to identify functional modules in which to compartmentalize the model. Rate your current confidence level at accomplishing this task on a scale of 1-5 (5 being "extremely high confidence").

8. Would you feel comfortable picking up a book(s), researching journals, and integrating information across multiple length scales to set up a modeling problem with which you have a strong interest and knowledge of the underlying system? Rate your current confidence level at accomplishing this task on a scale of 1-5 (5 being "extremely high confidence").

9. Suppose you have data on 100 different components that are identified for the progression of prostate cancer. You know that the capabilities of your computational resources limit you to no more than 20 components. Thus, you have to decide how you draw your system boundary such that the smaller subsystem is going to best address the question of interest. Rate your current confidence level at accomplishing this task on a scale of 1-5 (5 being "extremely high confidence").

10. Do you want to seek a job where you perform the following skills? In other words, which of the following skill sets would you prefer to use your future career?

a. Computer modeling 

b. Experimental analysis
c. High-throughput data analysis
d. Integrating heterogeneous datasets
e. None of these

Rank-order the skills that you would be most interesting in using in your future career. Fill in numbers 10-14 with one of the letters above, where 10 is your top preference, and 14 is your lowest preference.

In the second round of the survey given January 2009, two questions were added:

\section{How much does your Capstone project involve computational systems modeling and/or analysis? Rate your answer on a scale of 1-5 (5 being extensive, 1 being none). \\ 16. How do you feel that your ability to build and/or validate computational models has changed since last August? Rate your answer on a scale of 1-5 (5 being greatly improved, 1 being gotten much worse).}

For the results presented questions $10-14,1 \rightarrow 5$ corresponds to "very low preference" to "very high preference" for a job involving the skill in question. (All other scores are as indicated on the survey questions.) Table 1 below summarizes the weighted averages of the scores for all 14 questions in Round 1 and all 16 questions in Round 2 of the survey. In both surveys, the results were split into two populations: students who were enrolled (or who had already taken, in the case of the 2009 survey) the Systems Bioengineering Modeling and Experimentation Course. Results were compared between students enrolled in the course (i.e. just before taking the course and after completing it), although given the smaller sample sizes, only one question (Q7) showed a statistically significant difference (Wilcoxon $P<0.05$ ). In fact, this score, pertaining to the students' comfort level in teaching someone else how to build a model, actually decreased after taking the course. We believe that this difference is likely due to a greater appreciation of what is required to properly build a model and thus student overconfidence before taking the class. The second column of $P$-values (right-most column of Table 1) corresponds to the difference in the "after" survey only (i.e. January) between students who had taken the course and those who had not. Dark gray shading reflects a Wilcoxon $P<0.01$, and lighter shading $P<0.05$.

Students who took the course showed significantly higher comfort levels compared to those who did not take the course in ability to use a computational model to test drug targets in a metabolic network (even though all of them learn the basics of metabolic network analysis in a core course in the junior year), solving systems of ODEs (also covered in a core course in the curriculum), applying statistics to high-throughput data (even though they have all taken probability and statistics), and confidence in setting up a model form the literature and teaching someone else with a mathematics background how to do the same. However, given the small sample size of students who enrolled in the course and completed the survey $(N=8)$, we must be cautious in drawing strong conclusions. Given the high scores on most of these questions given by the students enrolled in the course before the course actually started (first column of the table), it is evident that students with a strong interest and self-perceived aptitude in modeling self-selected to enroll in the course. Students who took the systems course were also much more likely to use modeling extensively in their senior Capstone projects. 
Table 1. Results from survey instruments administered to students enrolled in the systems course in August $2008(N=6)$ and January 2009 after the course concluded $(N=8)$, as well as students who did not take the course ( $N=54$ and $N=43$, respectively).

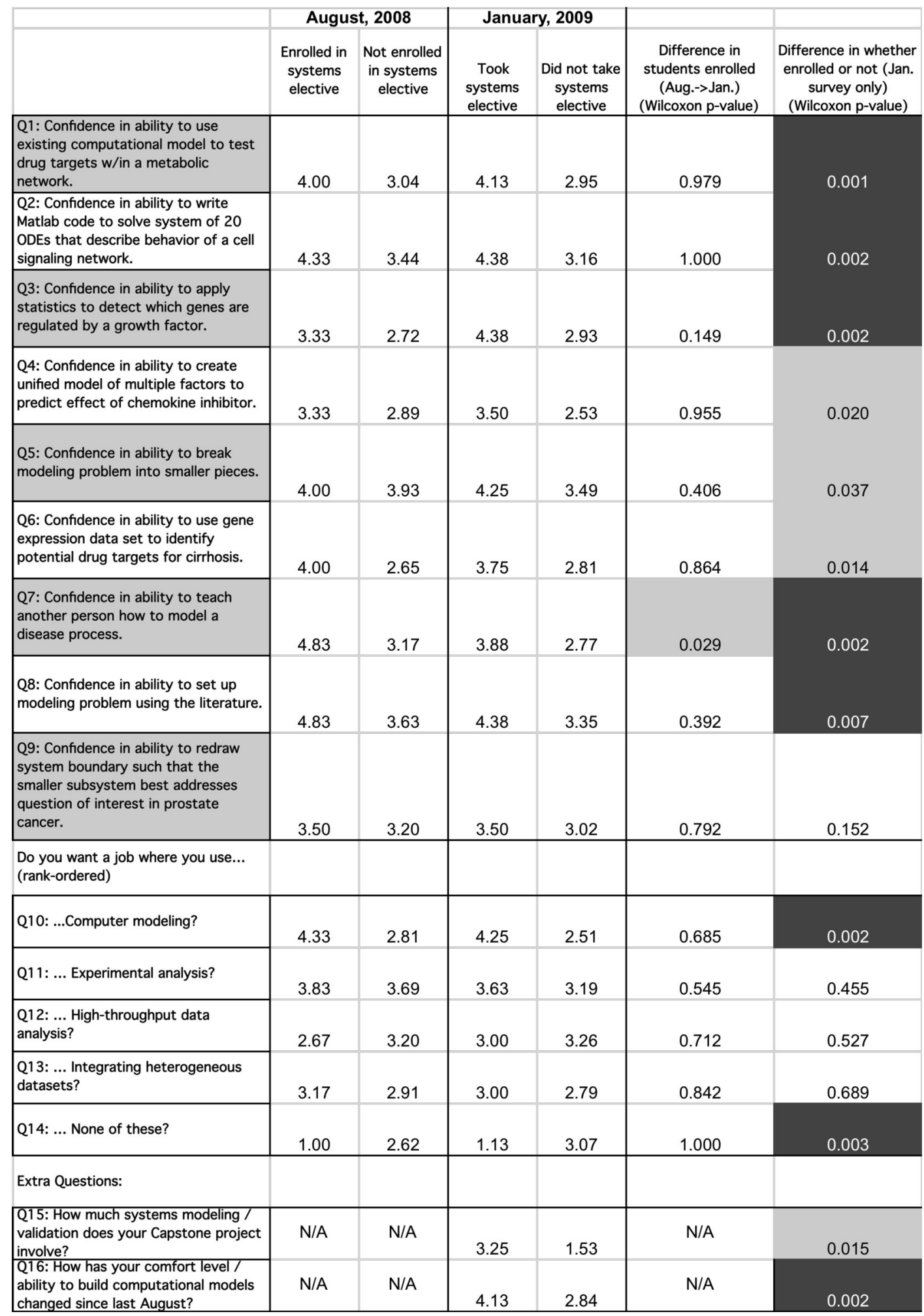


Students $(N=9)$ also completed anonymous course evaluations at the conclusion of the semester in December 2008. These evaluations were extremely positive, with the students rating the following on a 5-point Likert scale: "This course was challenging." $\rightarrow$ 4.56; "I learned a great deal in this course." $\rightarrow$ 4.89; and "Overall, this was a worthwhile course." $\rightarrow$ 4.89. Specific open-ended comments included:

- "I really enjoyed this class! Although it is a lot of work, but I was definitely learning a lot and would highly recommend it to everyone! Please try to keep this course for the future!"

- "Oral tests were a great idea, I am thankful I had an opportunity to get more practice presenting."

- "This was a very interesting and fun course. I enjoyed learning about new technologies and real applications of systems modeling, and the integrated laboratory experience was very beneficial. ... Overall, it was a great class, and I feel like I learned many valuable, practical skills. All of the instructors were very knowledgeable, and it was good to get to talk to them in more of a 1 on 1 setting. I would strongly encourage that this course be offered again next year."

- "Although this course required a lot of work both in the lab and at home, I though it was a great course with very interesting subject matter. I would definitely recommend it to future classes."

- "Overall, one of the best courses I have taken at the University."

The students appreciated the small class size and discussion-driven seminar style of many of the lectures, and they also appreciated the oral midterm exams. However, there were two main critiques of the course: 1) Students felt that the course could have used greater cohesion and linking of the various scales of modeling approaches. They agreed that we attempted to do this in the closing lectures, but they would have appreciated more linkages between intracellular, network, and multicellular modeling throughout the course. 2) Students would have appreciated more regular assignments to keep them on task, rather than just the three oral midterms and the final exam. They suggested short weekly homework problems or progress reports to achieve this purpose.

\section{Conclusions and Future Directions}

Overall, the first iteration of the Systems Bioengineering Modeling and Experimentation course taught at the University of Virginia was very successful based upon assessment of student learning throughout the semester, assessment of student confidence levels relative to the rest of the senior class (who did not take the elective), and anonymous student course evaluations. However, at this point it is difficult to draw strong comparative conclusions about the efficacy of the course because of the small sample size of the students who enrolled in the course $(N=9)$ and who participated in the surveys $(N=6$ and $N=8)$. It is also possible that students think that they possess more aptitude than they actually do, particularly before taking the course (thus leading to potentially inflated scores among those students in the August survey). A refined survey instrument that tests actual knowledge may better elucidate any real effect the course has relative to the rest of the students. 
In future iterations of the course, we will add lectures interspersed throughout the semester to better link the three modules conceptually and to help the students see how a true multi-scale biological model can be generated. There are also numerous tweaks to the experimental protocols to optimize both the quality of the results and the efficiency of the labs that we will institute in the next offering of the course. For example, in Module 3, we neglected to include enough time points measuring the vascular growth in the CAM assay to properly validate the agent-based model. We will also have to optimize some of the drug doses and time points in Modules 1 and 2 to see a greater response and achieve cleaner results.

In conclusion, we developed the Systems Bioengineering Modeling and Experimentation Course at U.Va. to teach our undergraduates about this rapidly emerging field within biomedical engineering. Industry is also becoming increasingly aware of the importance of systems biology as a field, and especially the process of using experimental data to iteratively build a model that has predictive value. Numerous biotechnology and pharmaceutical companies appreciate that most biological systems - and the interaction of therapeutics with those systems - can only be understood within the context of complex systems. ${ }^{31}$ However, we also recognize that BME is a broad field, and many students have interests in areas such as medical imaging, biomechanics, instrumentation, and tissue engineering. Thus, we have decided not to make this course a core part of the curriculum yet, particularly given the advanced level of the elective. But many aspects of the course are portable, such that if other programs wanted to adopt portions of the course (with or without the laboratory component) to their curriculum (whether in core courses or in electives), they would be able to do so. For instance, a molecular bioengineering course could use the Module 1 material in modeling signaling protein translocation into the nucleus in conjunction with relevant lecture material, or a smaller school with limited resources could adopt the computational aspects of one or more of the modules while using publicly available data, thereby obviating the need for the associated laboratories. The course described in this paper thus provides a starting point for using a module-based approach to teach the key concepts and approaches in systems biology.

\section{Acknowledgements}

We would like to thank Kitter Bishop for assistance with administering the survey instruments, and Will Guilford for advice on the assessment of the course. We would also like to acknowledge the National Science Foundation (DUE CCLI Proposal 0737415) for funding support for developing the course described in this paper.

\section{Bibliography}

1. Enderle, J., S. Blanchard, and J. Bronzino. 2005. Introduction to Biomedical Engineering. Elsevier Academic Press, Boston.

2. Lander, E.S. L.M. Linton, B. Birren, C. Nusbaum, M.C. Zody, J. Baldwin, K. Devon, K. Dewar, M. Doyle, W. FitzHugh, et al. 2001. Initial sequencing and analysis of the human genome. Nature, 409:860-921.

3. Venter, J.C., M.D. Adams, E.W. Myers, P.W. Li, R.J. Mural, G.G. Sutton, H.O. Smith, M. Yandell, C.A. Evans, R.A. Holt, et al. 2001. The sequence of the human genome. Science, 291:1304-1351. 
4. Mathew, J.P., B.S. Taylor, G.D. Bader, S. Pyarajan, M. Antoniotti, A.M. Chinnaiyan, C. Sander, S.J. Burakoff, and B. Mishra. 2007. From bytes to bedside: data integration and computational biology for translational cancer research. PLoS Computational Biology, 3(2):e12.

5. Tsien, R.Y. 2003. Imagining imaging's future. Nat. Rev. Mol. Cell Biol., Sep. Suppl:SS16-21.

6. Hood, L. and R.M. Perlmutter. 2004. The impact of systems approaches on biological problems in drug discovery. Nature Biotechnology, 22:1215-1217.

7. Bialek, W. and D. Botstein. 2004. Introductory science and mathematics education for 21st-century biologists. Science, 303:788-790.

8. Selinger, D.W., M.A. Wright, and G.M. Church. On the complete determination of biological systems. TRENDS in Biotechnology, 21:251-254.

9. Ideker, T. 2004. Systems biology 101—what you need to know. Nature Biotechnology. 22:473-475.

10. Lundstrom, K. 2006. Latest development in drug discovery on G protein-coupled receptors. Curr. Protein Pept. Sci., 7(5):465-470.

11. Gilman, A.G., M.I. Simon, H.R. Bourne, B.A. Harris, R. Long, E.M. Ross, J.T. Stull, R. Taussig, A.P. Arkin, M.H. Cobb, et al. 2002. Overview of the Alliance for Cellular Signaling. Nature, 420:703-706.

12. Horwitz, A.R., N. Watson, and J.T. Parsons. 2002. Breaking barriers through collaboration: the example of the Cell Migration Consortium. Genome Biol., 3(11): comment2001.

13. Kumar, N., B.S. Hendriks, K.A. Janes, D. de Graaf, and D.A. Lauffenburger. 2006. Applying computational modeling to drug discovery and development. Drug Discov. Today, 11:806-811.

14. Fox, S., S. Farr-Jones, L. Sopchak, A. Boggs, H.W. Nicely, R. Khoury, and M. Biros. 2006. High-throughput screening: update on practices and success. J. Biomol. Screen., 11:864-869.

15. Saucerman, J.J., and A.D. McCulloch. 2004. Mechanistic systems models of cell signaling networks: a case study of myocyte adrenergic regulation. Progress in Biophysics and Molecular Biology, 85(2-3):261-278.

16. Garber, K. 2004. Genomic medicine: gene expression tests foretell breast cancer's future. Science, 303:17541755.

17. Reed, J.L., I. Famili, I. Thiele, and B.O. Palsson. 2006. Towards multidimensional genome annotation. Nature Reviews Genetics, 7(2):130-41.

18. Davidson, E.H. 2006. The Regulatory Genome: Gene Regulatory Networks In Development And Evolution. Elsevier Academic Press, Boston.

19. Papin, J.A., T. Hunter, B.O. Palsson, and S. Subramaniam. 2005. Reconstruction of large-scale cellular signaling networks and analysis of their properties. Nature Reviews Molecular Cell Biology, 6:99-111.

20. Bild, A.H., G. Yao G, J.T. Chang JT, Q. Wang, A. Potti, D. Chasse, M.B. Joshi, D. Harpole, J.M. Lancaster, A. Berchuck, J.A. Olson Jr., J.R. Marks, H.K. Dressman, M. West, and J.R. Nevins. 2006. Oncogenic pathway signatures in human cancers as a guide to targeted therapies. Nature, 439:353-357.

21. Peirce S.M. and T.C. Skalak. 2003. Microvascular remodeling: a complex continuum spanning angiogenesis to arteriogenesis. Microcirculation, 10:99-111.

22. Nordlie M.A., L.E. Wold, B.Z. Simkhovich, C. Sesti, and R.A. Kloner. 2006. Molecular aspects of ischemic heart disease: ischemia/reperfusion-induced genetic changes and potential applications of gene and RNA interference therapy. $J$ Cardiovasc Pharmacol Ther, 11:17-30.

23. Taylor A., W.L. Murfee, and S.M. Peirce. 2007. EphB4 expression along adult rat microvascular networks: EphB4 is more than a venous specific marker. Microcirculation, 14:253-67.

24. Peirce, S.M., T.S. Skalak, and J.A. Papin. 2006. Multi-scale systems integration from cells to tissues: coupling intracellular network analysis with tissue-patterning simulations. IBM Journal of Research and Development, 50(6): 601-615.

25. Peirce S.M., E.J. Van Gieson, and T.C. Skalak. 2004. Multicellular simulation predicts microvascular patterning and in silico tissue assembly. FASEB Journal, 18: 731-733.

26. Barnhill, R.L. and T.J. Ryan. 1983. Biochemical modulation of angiogenesis in the chorioallantoic membrane of the chick embryo. J. Invest. Dermatol. 81:485-488.

27. Dusseau, J.W., P.M. Hutchins, and D.S. Malsaba. 1986. Stimulation of angiogenesis by adenosine on the chick chorioallantoic membrane. Circulation Research, 59:163-170.

28. Ribatti, D., A. Vacca, L. Roncali, and F. Dammacco. 1996. The chick embryo chorioallantoic membrane as a model for in vivo research on angiogenesis. Int. J. Dev. Biol., 40:1189-1897.

29. Bloom, B.S. \& D.R. Krathwohl. 1956. Taxonomy of educational objectives: The classification of educational goals, by a committee of college and university examiners. Handbook 1: Cognitive domain. New York, Longmans. 30. Krathwohl, D.R. 2002. A revision of bloom's taxonomy: An overview. Theory into Practice, 41 (4), 212-218. 31. Mack, G.S. 2004. Can complexity be commercialized? Nature Biotechnology, 22:1223-1229. 